SESSION 4

FRIENDLY DEBATE:

RELATION OF QUIET SUN TO ACTIVE REGIONS 


\title{
STRONG AND WEAK MAGNETIC FIELDS: NATURE OF THE SMALL-SCALE FLUX ELEMENTS
}

\author{
JAN OLOF STENFLO \\ Institute of Astronomy, ETH-Zentrum, CH-8092 Zurich, Switzerland
}

\begin{abstract}
The 20 year old conclusion that more than $90 \%$ of the magnetic flux recorded by magnetographs in quiet and active regions is in strong-field, $\mathrm{kG}$ form has recently been supported by direct observations of Zeeman splitting in the infrared (near $1.6 \mu \mathrm{m}$ ). The magnetic and thermodynamic properties of the $\mathrm{kG}$ fluxtubes have been explored in considerable detail over the past decade, in particular through Fourier transform spectrometer observations combined with Stokes inversion procedures. The weak fields have proven to be much more elusive, and it was not until last year that the first definite identifications of intrinsically weak (less than $\mathrm{kG}$ ) fields in the low photosphere could be made, using the Stokes $V$ profiles of an infrared line pair.
\end{abstract}

\section{EARLY INDICATIONS OF STRONG FIELDS: SOME PERSONAL REFLECTIONS}

My own experience with the sun's magnetic field started with an observing program that I carried out at the Crimean Astrophysical Observatory in the summer of 1965. Severny's (1965) work had highlighted the inhomogeneous structure of the solar magnetic field, and Alfven (1967) had argued that the field must be filamentary (due to the pinch effect of electric currents). With my observing program I wanted to explore how the inhomogeneous nature of the field affects magnetograph observations and the conclusions we try to draw from them.

It was well known that active regions harbour strong, intermittent fields, so my whole attention was focused on very quiet regions far from any activity centers. I mapped the longitudinal magnetic field of the same quiet regions using different sizes of the scanning aperture (height of spectrograph slit) to explore how the apparent field strengths depend on the spatial resolution used (down to about 2 arcsec resolution). The results, published in an obscure Swedish journal (Stenflo, 1966), showed that the measured field strengths increase rapidly with increasing spatial resolution, as would be expected if the magnetic flux exists in the form of discrete clumps of a size much smaller than the smallest scanning aperture used. There was however no means of telling how strong the field would be if infinite spatial resolution could be used.

From this initial experience it was clear to me that the interpretation of magnetograms is far from straightforward. The word "magnetograph" may 
actually be misleading, since it suggests that the output of such an instrument is the magnetic field. Instead it is of course a map of the circular polarization at a fixed wavelength. The relation between polarization and magnetic field depends on many different, interconnected physical parameters governing the atmospheric structure in the region under study, like temperature, opacity, Doppler shifts, height gradients, etc. In a theoretical paper published in a still more obscure Swedish journal (Stenflo, 1968) I explored in terms of a multi-component model the general problem how magnetograph observations are affected if we have a distribution of physical parameters within the resolution element. The two-component case, with one magnetic and one non-magnetic component, was considered as a special case, but at that time there was no empirical indication that this special case would be a useful approximation.

In the late 1960's several other pieces of evidence for spatially unresolved strong fields in quiet regions began to emerge. Sheeley (1967) found moderately strong fields (with an apparent strength of a few hundred $G$, which at that time was considered as very strong) in the quiet network at locations of line "gaps" in his spectrograms. By comparing lines of different excitation potential Chapman and Sheeley (1968) could conclude that the gap phenomenon was largely due to temperature weakenings of the lines, implying a temperature enhancement in the flux elements with respect to their surroundings at equal optical depth. The "invisible" flux elements of the quiet network were thus not dark microspots but were of the same nature as solar faculae.

A very influential piece of work in the late 1960's was the paper of Harvey and Livingston (1969), who made scatter plots of the apparent field strengths recorded by the Kitt Peak magnetograph simultaneously in two different spectral lines. They found well defined regression lines with a slope differing from unity, but interpreted these discrepancies exclusively in terms of temperature effects, which was natural to do at that time in view of the results of Chapman and Sheeley (1968). Furthermore their choice of line pairs did not allow the temperature effects to be separated from other possible effects. Nevertheless this work was a forerunner to the later introduction of the line-ratio method as a means of diagnosing the spatially unresolved magnetic field. The well-defined regression relations also gave a good indication that a two-component model approach would work for the interpretation of such data.

When later the line-ratio work (see below) showed that more than $90 \%$ of the total magnetic flux seen by magnetographs is in $\mathrm{kG}$ form, implying a global magnetic filling factor of only about $1 \%$, two types of questions arose: (1) What are the properties and origins of the kG flux elements? (2) What is the nature of the field in the remaining $99 \%$ of the photosphere? Due to space limitations, we will only deal with the fluxtube problem here. My views on the second problem area, which concerns the mixed-polarity weak fields, have been summarized in Stenflo $(1989,1992 \mathrm{a}, \mathrm{b})$. Recently a quantitative identification of the turbulent magnetic field was made possible by analysis of the Hanle effect in the Sr I $4607 \AA$ line (Faurobert-Scholl, 1992).

\section{THE LINE-RATIO METHOD}

Around 1970 much evidence had accumulated, which indicated that most of the magnetic fine structure was still far from spatially resolved. As a resolution of 
close to one arcsec had been reached, and great improvements in the near future could not be expected due to the seeing limitations imposed by the earth's atmosphere, indirect methods had to be found that could provide an answer to the question what the field would look like if it could be observed with infinite spatial resolution. The spatial-resolution limit may be overcome by making use of additional information in the spectral domain. Such methods are common in other areas of astrophysics, where a great deal of information on apparently point-like objects can be gained by analysing their spectra.

If we consider the solar flux elements as isolated point-like objects, their spectra (without polarization analysers) are usually drowned by the superposed radiation from their "non-magnetic" surroundings. It is as if we would observe the stars at daylight. In circular polarization, however, the surroundings are "dark", the background goes away. It is therefore imperative for quantitative analysis to base network and facular models on polarization data to avoid this contamination problem.

The observed polarization depends on the stratification of the physical parameters along the line of sight and is thus a function of many parameters that are coupled to each other in a highly non-linear fashion. The basic idea behind the line-ratio method is to select two spectral lines that are affected identically by all the physical parameters except one, e.g.the field strength, thereby allowing the effects of this single parameter to be isolated from all the other effects. There are not many line pairs that satisfy this criterion, so the choice of lines has to be made with great care and awareness of the possible effects.

Fortunately there exists a quite "clean" line ratio that isolates the field strength (in the form of Zeeman saturation) from the other effects. The two lines are Fe I 5250.2 and $5247.1 \AA$, which both belong to multiplet No. 1 of neutral iron, have the same equivalent widths and excitation potentials, but differ in their Landé factors ( 3.0 and 2.0, respectively). This line ratio is illustrated in Fig. 1.

The 5250/5247 magnetic line ratio was first introduced in an observing run at Kitt Peak in September 1971 (Stenflo, 1973). A very quiet region right at the center of the solar disk was scanned. Of the 1943 spatial points sampled by the $2.4^{\prime \prime} \times 2.4^{\prime \prime}$ aperture, not a single one indicated an apparent field strength (average flux density) in excess of $50 \mathrm{G}$, and the majority of the values were below $3 \mathrm{G}$. Nevertheless the line-ratio information implied that the actual, true field strengths of the spatially unresolved flux elements contributing to the apparent fluxes were in the $1-2 \mathrm{kG}$ range.

Figure 1 gives a summarizing overview of the 5250/5247 magnetic line ratio and illustrates the physics behind it. The scatter-plot diagram to the upper left, from Frazier and Stenflo (1978), is based on magnetograph mapping of an area of the sun, sampling thousands of spatial points while keeping the exit spectral windows in the wings of the two lines fixed. The diagram to the upper right, on the other hand, from Stenflo and Harvey (1985), is based on spectral Stokes $I$ and $V$ recordings, similar to that of the lower diagram, for a relatively small number of spatial points on the sun. The line ratio is then based on the Stokes $V$ amplitudes of the two lines. This Stokesmeter approach results in a considerably higher $\mathrm{S} / \mathrm{N}$ ratio in the determined line ratio, but the statistical sample is much smaller. The scatter around the regression line in the upper left diagram is consistent with the measured noise level. There is no need for intrinsic solar 

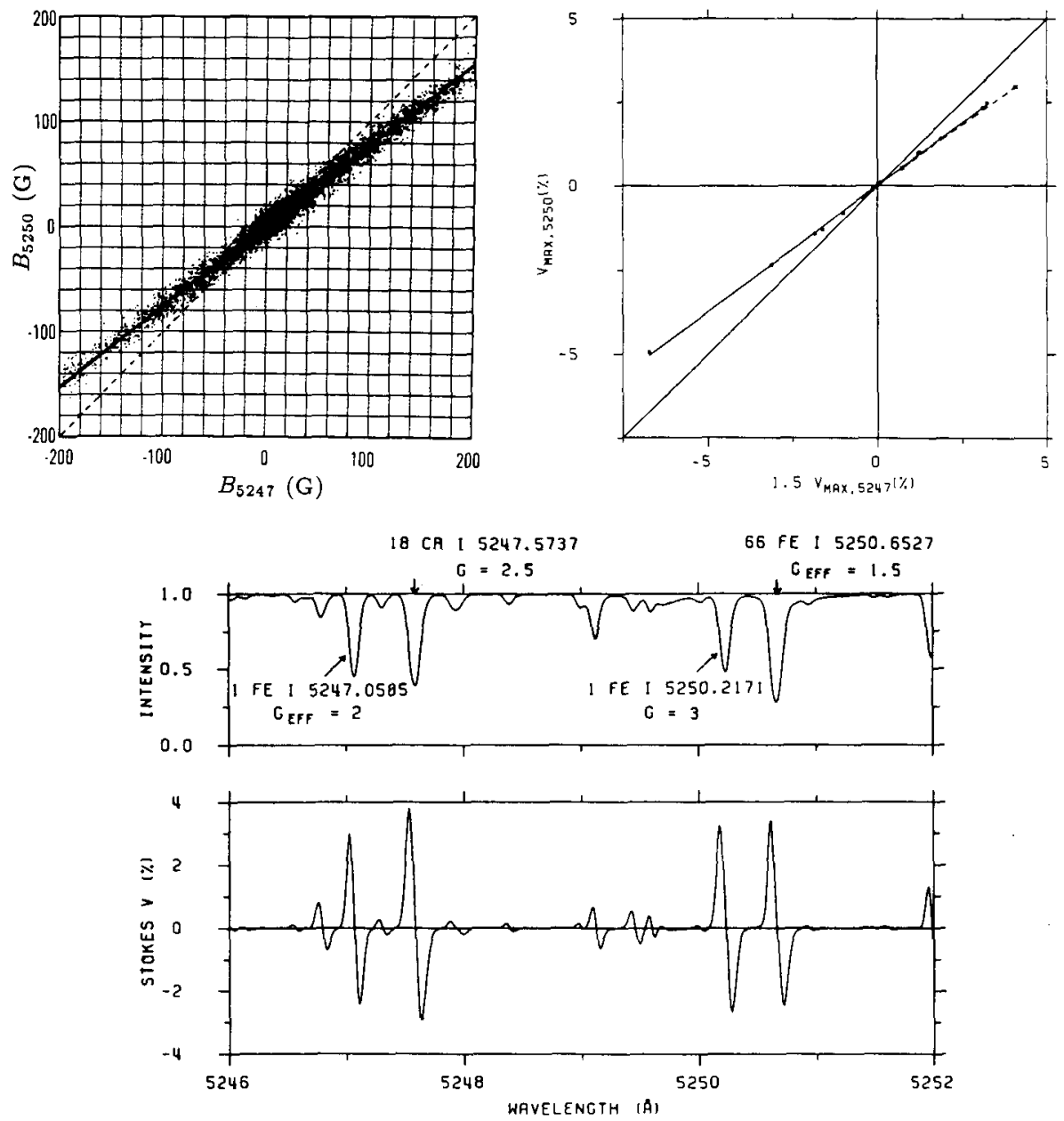

Fig. 1. Illustration of the line-ratio method (see text).

scatter to explain the diagram, although some degree of solar scatter of course cannot be ruled out.

The spectral recording in the lower diagram, from Stenflo et al.(1984), was made with the Fourier transform spectrometer (FTS) of the Kitt Peak McMath telescope. If the fields were intrinsically weak $(\leqslant 0.5 \mathrm{kG})$, then the relation between magnetic flux and circular polarization would be linear, and the ratio between the Stokes $V$ amplitudes of the 5250.2 and 5247.1 $\AA$ lines would be $3: 2$, the ratio between their Landé factors. Visual inspection of the spectrum immediately reveals that the ratio is considerably smaller than this value, which is naturally explained in terms of Zeeman saturation, i.e., deviation from a linear relation that sets in when the Zeeman splitting is no longer small in comparison with the line width. It is a differential effect - it mainly affects the $5250.2 \AA$ line with the large Landé factor. 
With the availability of full line profiles one is of course not limited to extracting the ratio between the Stokes $V$ amplitudes, but one can determine the Stokes $V$ line ratio as a function of $\Delta \lambda$, the distance from line center. The Zeeman saturation not only suppresses the amplitude but widens the profile and has a special spectral signature that is fully verified by the FTS profile analysis.

The thermal effects can be seen by inspecting the $5250.7 \AA$ line. Although its Landé factor is only 1.5, its Stokes $V$ amplitude is as large as that of the $5250.2 \AA$ line. This is largely due to the smaller temperature weakening of the $5250.7 \AA$ line (it has a higher excitation potential), although a significant part of the explanation is in terms of the Zeeman saturation of the $5250.2 \AA$ line. We thus see that a combination of all three lines mentioned provides both field strength and temperature information: having derived the field strength from the magnetic line ratio, it is possible to extract the temperature information from the $5250.7 \AA$ line.

In a scatter-plot like that of the diagram to the upper left of Fig. 1 the slope of the regression line is much determined by the points with the largest fluxes, although the majority of the sampled points represent tiny flux values, crowded around the origin of the diagram. The question then arises whether these small fluxes may represent fields that are intrinsically weak, or whether they also are due to unresolved $\mathrm{kG}$ fields, although with very small filling factors. Due to the large number of small-flux points, they contribute to a significant fraction of the total flux recorded in a magnetogram.

If $B_{1, i}$ is the apparent magnetic flux density in line no. 1 at spatially sampled point no. $i$, while $B_{2, i}$ is the corresponding value in line no. 2 , we may account for the accumulated contributions from all the small-flux points by adding up the absolute values of the apparent flux densities and forming the ratio

$$
\sum_{i}\left|B_{1, i}\right| / \sum_{i}\left|B_{2, i}\right|
$$

between these accumulated apparent fluxes. If this accumulated flux ratio is the same as the slope of the regression line for the same data set, then one may infer that all the fluxes, also those that are apparently very weak, are due to strong, $k \mathrm{G}$ fields. Such arguments were used by Howard and Stenflo (1972) and Frazier and Stenflo (1972) to conclude that more than $90 \%$ of the total flux recorded by magnetographs with a spatial resolution of a few arcsec or larger (e.g. the Kitt Peak and Mt Wilson magnetographs) is due to strong fields. The value of $90 \%$ was determined by the error bars of the observations. The accumulated flux ratio and the slope value were found to agree within a certain error margin.

\section{FTS POLARIMETRY AND FLUXTUBE MODELING}

The advent of polarimetry with an FTS (Fourier transform spectrometer) brought a new dimension to the diagnostics of solar magnetic fields, since fully resolved line profiles of hundreds of simultaneously recorded spectral lines became available for analysis, in contrast to two fixed wavelength bands in the early line-ratio work with Babcock-type magnetographs. With FTS data it is no longer necessary to work with line pairs that may isolate the effects of one physical parameter, but one may choose combinations of any number of lines, with the only requirement that the chosen lines should respond as 
differently as possible to perturbations of the atmospheric structure. With the set of observables suitably chosen, the Stokes inversion problem becomes numerically stable, so that empirical fluxtube models giving the height variation of all the magnetic and thermodynamic parameters may be constructed. The hundreds of polarized line profiles that have to be reproduced by any fluxtube model provide a rich set of constraints on any such model. The circumstance that it is at all possible to find physically self-consistent models that satisfy all the new constraints that have so far been used is in itself evidence for the correctness of the concept of discrete, $\mathrm{kG}$ fluxtubes in pressure equilibrium with their surroundings.

The FTS data, a small example of which was illustrated in Fig. 1, have provided the basis for a great activity in model building of solar magnetic fluxtubes (cf. Solanki, 1990). The facular models of the past (mainly before the mid 1970's), which were based on Stokes $I$ observations with an unknown filling factor, have thereby become obsolete. The reason for basing the models on the Stokes $V$ information is to get rid of the massive contamination from surrounding radiation, since the Stokes $V$ signal gets its contributions exclusively from the magnetic elements, regardless of how small and unresolved these elements are, i.e., regardless of how small the magnetic filling factor is.

Two qualitatively different modeling approaches have been used: (1) Inversion of the observed Stokes data. This leads to fully empirical but physically consistent (satisfying the requirement of lateral and vertical pressure balance, flux conservation, etc.) models. (2) Theoretical modeling. One starts from the MHD equations coupled with equations for radiative and convective energy transport, and derives the fluxtube structure that is a consequence of these equations. This generally requires massive number crunching on supercomputers, but so does Stokes inversion when it is performed at a sufficiently sophisticated level (a level that has been reached since a few years).

The agreement between the theoretical and empirical models is rather good, but there are differences in detail, suggesting that certain physics is missing in the energy equation used in the theoretical models. In particular we expect wave heating to significantly affect the thermodynamics of the fluxtubes, but this has not yet been modeled in this context.

Figure 2 illustrates some modeling results. The left diagram gives the height variation of the field strength, based on Stokes inversion by Zayer et al.(1990) (the different curves refer to different filling factors). This fieldstrength variation implies that the fluxtubes are highly evacuated, with empirically determined values for the plasma $\beta$ (ratio between gas and magnetic pressure) of typically 0.3 . The diagram to the right, from Keller et al.(1990), shows the temperature structure of four models in comparison with the temperature of the outside, undisturbed reference atmosphere (solid line). Two of the models are empirical, due to Keller et al.(1990), represented by the two dashed curves that run almost parallel to each other. The upper, hotter curve represents a network element with small magnetic filling factor, the lower curve a facular element with larger filling factor. The two remaining curves represent theoretical models. The dashed-dotted curve is due to GrossmannDoerth et al.(1989), the steeper, dashed curve is due to Steiner and Stenflo (1990).

Although different in detail, the empirical and theoretical models share a common property: they are cooler than their surroundings in the deep 

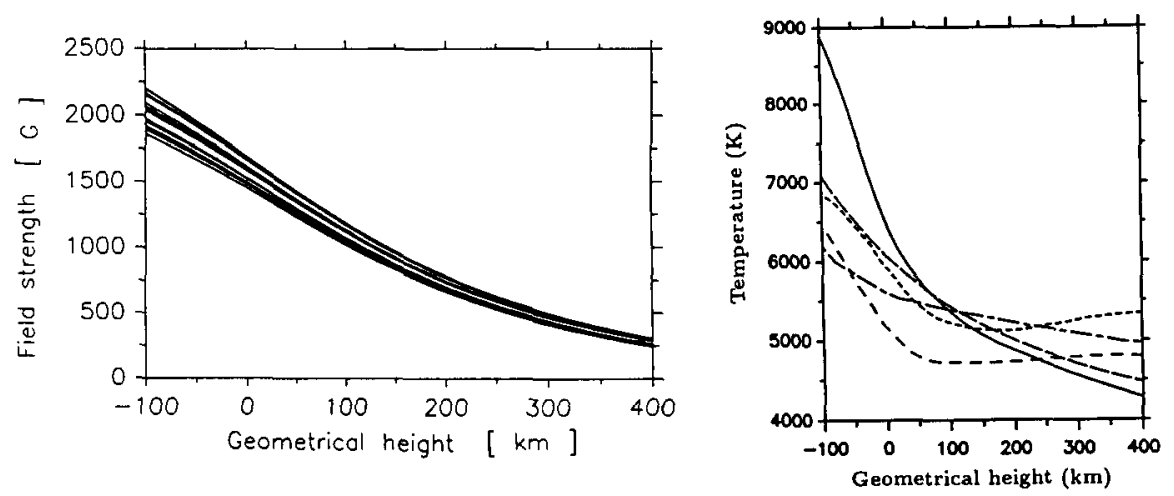

Fig. 2. Empirically derived height variations of fluxtube field strength (left) and temperature (right), together with two theoretical temperature models (see text).

atmosphere, hotter higher up. In the absence of wave heating the increased temperature in the higher layers is due to $2-\mathrm{D}$ radiative energy transfer by radiation from the deeper hot walls of the fluxtube illuminating the higher layers of the fluxtube interior.

In spite of the network elements being cooler than their surroundings deep down, they turn out to be hotter everywhere at equal optical depth, which is what counts for their optical appearance on the sun. Also, in the continuum radiation at disk center, the fluxtubes of the quiet network appear as bright features. The facular elements representing larger filling factors are however systematically cooler, and appear dark in the disk-center continuum. This fillingfactor dependence determining whether a feature appears bright or dark in the continuum has led to considerable confusion in the past, but has recently been clarified by the work of Solanki and Brigljević (1992). Recently observations using speckle polarimetry have resolved fluxtubes with a diameter of about $200 \mathrm{~km}$ and a continuum brightness exceeding $30 \%$ of the surroundings near the center of the solar disk (Keller, 1992 and these proceedings).

\section{INFRARED DIAGNOSTICS}

The great potential of magnetic-field diagnostics in the infrared was demonstrated by the pioneering work of Harvey and Hall (1975), who found $\mathrm{kG}$ fields in quiet regions by directly measuring the Zeeman splitting of the sigma components of an Fe I line at a wavelength of $1.5649 \mu \mathrm{m}$. This provided independent confirmation of the line-ratio results on the field strengths in the quiet network, but only few positions on the solar disk were sampled. Since the Zeeman splitting increases as $\lambda^{2}$ whereas the line width increases approximately with $\lambda$, the Zeeman splitting in the fluxtubes is complete for the $1.5649 \mu \mathrm{m}$ line, which has a Landé factor of 3.0. The field strength can then be obtained by measuring the splitting directly.

Another advantage of the $1.6 \mu \mathrm{m}$ region is that it represents the opacity minimum of the solar spectrum, allowing us to see to the deepest layers of the solar atmosphere, where the magnetic field is most concentrated. It is only 

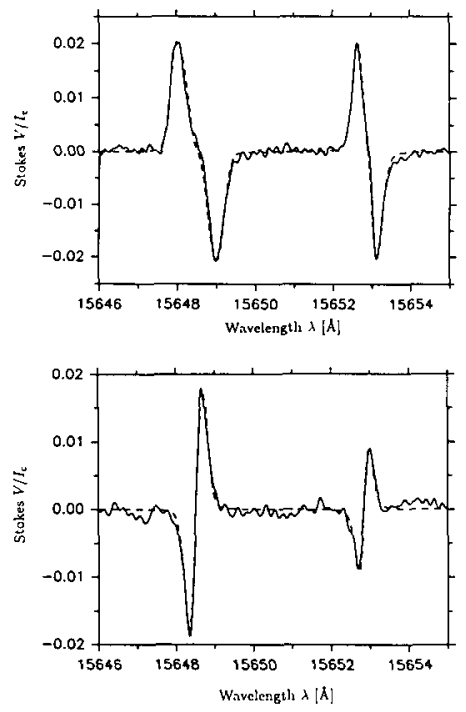

Fig. 3. Observed (solid) and synthetic (dashed) Stokes $V$ profiles of an infrared line pair (see text).

recently that this spectral region has begun to be systematically exploited, first by FTS polarimetry (Stenflo et al. , 1987), later with the McMath spectrograph (Livingston, 1991; Rüedi et al. , 1992) and Near-Infrared Magnetograph (Rabin, $1992 \mathrm{a}, \mathrm{b})$, as will be discussed below.

Another infrared region of great interest and future potential is the one around $12 \mu \mathrm{m}$. Brault and Noyes (1983) first discovered emission lines with remarkably large Zeeman splitting in this region. Of particular interest is a $\mathrm{Mg} I$ line at $12.32 \mu \mathrm{m}$, which has in recent years been explored polarimetrically by Hewagama etal.(1991), cf. the review by Deming in these proceedings. Although most $12 \mu \mathrm{m}$ work has been done in sunspots, some recordings in quiet regions indicate that the field is intrinsically weak $(300-500 \mathrm{G})$ there, which at first glance may appear as a contradiction to the $\mathrm{kG}$ field strengths found with other methods.

The low value of the field strength is however a natural consequence of the difference in height of formation of the spectral lines used. According to Carlsson et al. (1992) the Mg I $12.32 \mu \mathrm{m}$ emission line is formed in the upper photosphere at a height where the field strength in the empirical fluxtube models of Fig. 2 has fallen from close to $2 \mathrm{kG}$ at the level of continuum formation to $300-500 \mathrm{G}$. The $12 \mu \mathrm{m}$ observations are thus consistent with the $\mathrm{kG}$ fluxtubes diagnosed with other spectral methods, but detailed non-LTE radiative transfer calculations of $\mathrm{Mg}$ I formation in the fluxtube atmosphere will have to be done to allow this infrared region to be used quantitatively to constrain the fluxtube structure, in particular the height divergence and canopy formation.

Detailed quantitative analysis with Stokes inversion has already been done for the Stokes observations in the $1.6 \mu \mathrm{m}$ region (Zayer et al. , 1989, 1990; Rüedi et al. , 1992). Due to the large Zeeman splitting, a distribution of field strengths within the resolution element or along the line of sight causes considerable Zeeman broadening of the line profiles. To separate this Zeeman broadening from 


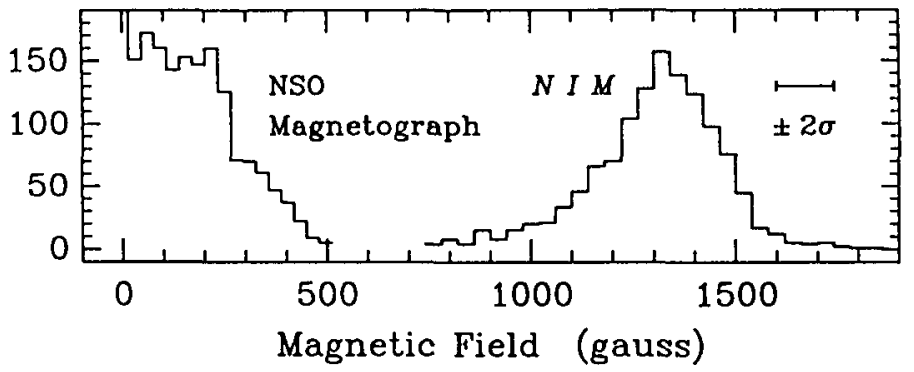

Fig. 4. Results from the Near-Infrared Magnetograph (Rabin, 1992a,b). The two histograms represent the apparent flux densities (left) and the true field strengths (right).

macroturbulent line broadening it is indispensible to use a line-ratio approach and combine the FeI $1.5649 \mu \mathrm{m}$ line with another Fe I line with a substantially smaller Lande factor. When this is done it is found that the entire Zeeman broadening can be naturally explained by the line-of-sight variation due to the height divergence of the field, without any need for a spread in field-strength values perpendicular to the line of sight.

The application of an infrared line ratio is illustrated in Fig. 3, from Rüedi et al. (1992). The two Fe I lines have Landé factors of 3.0 and 1.53 , respectively. The solid curves represent the observed Stokes profiles, the dashed curves the synthetic ones derived from the fluxtube model that fits the observations. In the upper diagram the field strength at $z=0\left(\tau_{5000}=1\right)$ is $1.52 \mathrm{kG}$, and the Zeeman splitting is complete for both Fe I lines. Their polarization amplitudes are therefore similar, but the line with the larger Landé factor is significantly broader due to differential Zeeman broadening. In the lower diagram the field strength at $z=0$ is much smaller $(0.75 \mathrm{kG})$, with less Zeeman broadening and with suppression of the polarization amplitude of the line with the smaller Landé factor, since this line is not fully split for these weaker fields.

The lower diagram of Fig. 3 represents the first identification of truly weak (below kG) fields in the lower photosphere. Several other examples of weak fields were found with this technique, but still about $90 \%$ of the total magnetic flux of the sample analysed was found to be due to fluxtubes with field strengths in excess of $1 \mathrm{kG}$.

A new dimension of field-strength diagnostics has been introduced with the Near-Infrared Magnetograph (NIM) of Rabin (1992a,b), which allows two-dimensional mapping of the intrinsic field strength across the solar surface, by measuring the Zeeman splitting of the Fe I $1.5649 \mu \mathrm{m}$ line recorded spectrographically with an InSb detector array. Figure 4 gives an example of initial results with NIM. Two histograms of the same solar area are shown, one for the true field strengths from NIM, the other for the apparent field strengths from the NSO magnetograph, which like all magnetographs belongs to the category of flux-measuring instrument. We see that the apparent flux densities have little to do with the true field strengths, most of which are found to be between 1.1 and $1.5 \mathrm{kG}$. The distribution has only a weak tail below $1 \mathrm{kG}$. The bulk of the flux is in excess of $1 \mathrm{kG}$. This represents a modern confirmation of the $20 \mathrm{yr}$ old result that more than $90 \%$ of the flux seen by magnetographs is in $\mathrm{kG}$ form (Howard and Stenflo, 1972; Frazier and Stenflo, 1972). 


\section{REFERENCES}

Alfvén, H. 1967, in Magnetism and the Cosmos, eds. W.R. Hindmarsh, F.J. Lowes, P.H. Roberts, S.K. Runcorn, Oliver \& Boyd, London/Edinburgh, pp. 246-261.

Brault, J.W., Noyes, R.W. 1983, Ap. J., 269, L61.

Carlsson, M., Rutten, R.J., Shchukina, N.G. 1992, Astr. Ap., 253, 567.

Chapman, G.A., Sheeley, N.R. Jr. 1968, Solar Phys., 5, 442.

Faurobert-Scholl, M. 1992, Astr. Ap., in press.

Frazier, E.N., Stenflo, J.O. 1972, Solar Phys., 27, 330.

Frazier, E.N., Stenflo, J.O. 1978, Astr. Ap., 70, 789.

Grossmann-Doerth, U., Knölker, M., Schüssler, M., Weisshaar, E. 1989, in Solar and Stellar Granulation, eds. R.J. Rutten, G. Severino, Kluwer, Dordrecht, p. 481.

Harvey, J.W., Livingston, W. 1969, Solar Phys., 10, 283.

Hewagama, T., Deming, D., Jennings, D.E., Osherovich, V., Wiedemann, G., Zipoy, D., Mickey, D.L., Garcia, H. 1992, Ap. J., , in press.

Howard, R., Stenflo, J.O. 1972, Solar Phys., 22, 402.

Keller, C.U. 1992, Nature, 359, 307-308.

Keller, C.U., Solanki, S.K., Steiner, O., Stenflo, J.O. 1990, Astr. Ap., 233, 583-597.

Livingston, W. 1991, in Solar Polarimetry, ed. L.J. November, NSO, Sunspot, NM, pp. 356-360.

Rabin, D. 1992a, Ap. J., 390, L103-L106.

Rabin, D. 1992b, Ap. J., 391, 832-844.

Rüedi, I., Solanki, S.K., Livingston, W., Stenflo, J.O. 1992, Astr. Ap., 263, 323-338.

Severny, A.B. 1965, Astron. Zh., 42, 217.

Sheeley, N.R. Jr. 1967, Solar Phys., $1,171$.

Solanki, S.K. 1990, in Solar Photosphere: Structure, Convection and Magnetic Fields, ed. J.O. Stenflo, IAU Symp., 138, 103-120.

Solanki, S.K., Brigljević, V. 1992, Astr. Ap., 262, L29-L32.

Steiner, O., Stenflo, J.O. 1990, Solar Photosphere: Structure, Convection and Magnetic Fields, ed. J.O. Stenflo, IAU Symp., 138, 181-184.

Stenflo, J.O. 1966, Arkiv Astron., 4, 173-198.

Stenflo, J.O. 1968, Acta Univ. Lund. II No. 2 (=Medd. Lunds Astron. Obs. Ser. II No. 153).

Stenflo, J.O. 1973, Solar Phys., 32, 41-63.

Stenflo, J.O. 1989, Astron. Astrophys. Rev., 1, 3-48.

Stenflo, J.O. 1992a, in The Solar Cycle, ed. K.L. Harvey, PASP Conf. Ser., 27, $83-88$.

Stenflo, J.O. 1992b, in Proc. OSL Workshop on Electromechanical Coupling of the Solar Atmosphere, ed. D.S. Spicer, Amer. Inst. Phys., in press.

Stenflo, J.O., Harvey, J.W. 1985, Solar Phys., 95, 99-118.

Stenflo, J.O., Harvey, J.W., Brault, J.W., Solanki, S.K. 1984, Astr. Ap., 131, 333-346.

Stenflo, J.O., Solanki, S.K., Harvey, J.W. 1987, Astr. Ap., 173, 167-179.

Zayer, I., Solanki, S.K., Stenflo, J.O. 1989, Astr. Ap., 211, 463-475.

Zayer, I., Solanki, S.K., Stenflo, J.O., Keller, C.U. 1990, Astr. Ap., 239, 356366. 